PROTOCOL BFM-95 IN TREATMANT OF CHILHOOD ACUTE LYMPHOBLASTIC LEUKEMIA (ALL) AND LYMPHOBLASTIC LYMPHOMA (LL)

doi:10.1136/archdischild-2012-302724.1471

1,2E Bojadjieva, ${ }^{3} \mathrm{~K}$ Martinova. 'Department of Pediarics, University Goce Delchev, Stip; 2Department of Oncology and Haematology, University Children Hospital; ${ }^{3}$ University Children Hospital, Skopje, FYR Macedonia

Introduction ALL and LL as the most common malignant diseases in childhood are two related malignancies that are treated by the same protocols.

Aim The aim of the study is to see the outcome of the application of protocol BFM-95 in the treatment of ALL and LL in childhood in Macedonia in the period 2000-2007.

Method We analyzed the medical records of 88 pediatric patients treated at Department of Hematology and Oncology at the University Pediatric Clinic in Skopje, 69 of which with ALL and 19 with LL. Results The annual average registered patients with ALL was 8.6 and 2.4 patients with LL. At the end of the study $85.5 \%$ patients with ALL and $42.1 \%$ patients with LL were alive. Regarding the outcome of the examined patients there were statistically significant differences between the two groups $(p=0,0075)$. There is a strong correlation between the type of disease and the outcome of the disease in patients $(\mathrm{p}=0,00001)$. Relaps was registered in $7.3 \%$ patients with ALL and in $31.6 \%$ patients with LL, where the analysis showed that between the two studied groups there is statistically significant difference $(p=0,0079)$. There is a correlation between the type of disease and the occurrence of relapse ( $p=0,0149)$. According to Kaplan-Meier-survival curve, a 5 -year overall survival is $92.3 \%$ in the group with ALL and $43 \%$ in group with LL.

Conclusion Although these two related cancers are treated with the same protocols, with current therapeutic approaches the outcome is better for patients with ALL compared to patients with LL.

\section{ULTRASOUND IMAGING OF ABDOMINAL MALIGNANCIES IN NEONATES AND FETUSES}

doi:10.1136/archdischild-2012-302724.1472

1.21 Begun. 'Belarusian Research Center for Pediatric Oncology, Hematology and Immunology; ${ }^{2}$ Belarusian Medical Academy of Post-Graduate Education, Minsk, Belarus

Purpose To study cases of the diagnosis of main abdominal malignancies in infants in the neonatal period and to review the effectiveness of ultrasound for these pathology from the fetus.

Patients and Methods A retrospective analysis of clinical and anamnestic data of the first month of life of children with retroperitoneal neuroblastoma, hepatoblastoma, renal tumors, who were treated in a two Belarusian cancer centers for the period from 1995 to 2011 were made. In the study group were included 16 neonates: 9 - with neuroblastoma, 3 - with hepatoblastoma, 4 - with mesoblastic nephroma.

Results It is established that the primary method of prenatal and postnatal diagnosis of the main forms of malignant abdominal tumors in all cases were the ultrasound diagnostic study. No there were neonates with nephroblastoma in this study. The highest diagnostic efficacy of ultrasound in the perinatal period has been observed in cases of mesoblastic nephroma. Half of them were diagnosed prenatally. Value for prenatal and postnatal diagnosis for neuroblastoma was 1:8. Prenatal detection of hepatoblastoma in the observed cohort of infants was ineffective. Postnatal volume this tumor was $150-282 \mathrm{ml}$. This are were definitely a congenital tumor.

Conclusions Ultrasonography is a method of choice for initial imaging of the tumors in the neonatal period. Obviously, there are diagnostic reserves for increased efficiency of prenatal detection of malignant and potentially malignant abdominal tumors. The results can be useful when planning screening programs for children in the first year of life.

\section{PANCREATIC NEUROENDOCRINE TUMORS IN CHILDREN}

doi:10.1136/archdischild-2012-302724.1473

S Sayyahfar. Tehran University of Medical Sciences, Tehran, Iran

Objectives Neuroendocrine tumors of the pancreas are rare specifically in pediatric age group. We report a 3.5-year-old girl with four weeks of icterus and primary misdiagnosis of hepatitis A and final diagnosis of well differentiated low grade neuroendocrine carcinoma of the pancreas. With our best knowledge this is the first report of this tumor in under -5 -year-group.

We highlight the patient's presentation, examination, and management as well as review the characteristics of pancreatic neuroendocrine tumors.

Methods A retrospective case study of a 3.5-years-old girl with history of 4 weeks Jaundice, weight loss, hepatomegaly and positive total anti HAV antibody without fever, abdominal pain, vomiting, or palpable mass in abdominal examination.

Results IgM anti HAV, $\mathrm{HBs} \mathrm{Ag}$, and $\mathrm{HCV} \mathrm{Ab}$ were all negative except for IgG anti HAV. Abdominal CT scan with oral and IV contrast revealed an intermediate enhancing mass in pancreatic head suggesting hypervascular pancreatic mass lesion. Examination and immunohistochemical staining of the tumor revealed well differentiated neuroendocrine carcinoma with extension to duodenal wall and lymph node metastasis.

Conclusion Despite of low incidence, the pediatricians should be aware and consider tumors in differential diagnosis of jaundice in children specifically if prolonged. In addition total anti HAV Ab test for diagnosis of hepatitis A should be discouraged.

\section{ACUTE BRONCHIOLTIS? OR A THORACIC MASS IN AN INFANT}

doi:10.1136/archdischild-2012-302724.1474

'FB Çakır, ${ }^{2} \mathrm{D}$ Karacanoğlu, ${ }^{3 B}$ Bilgiç. 'Pediatric Hematology-Oncology Department; 2Pediatrics Department, Bezmialem Vakif University; ${ }^{3}$ Pathology Department, Istanbul University Istanbul Medical Faculty, Istanbul, Turkey

A 7-month old girl with the complaints of crying, cyanosis and breathing rapidly was brought to a hospital by her family. After taking nebulised salbutamol, the symptoms relieved. However, the symptoms reoccurred despite appropriate acute bronchiolitis treatment. She was hospitalized due to massive right thoracic infiltration in the chest x-ray. During evaluation, thoracal ultrasound showed a suspicious solid mass at the posterior mediastinum. Thorax computerised tomography revealed a right hemithoracal and posterior mediastinal gross solid mass. As the staging procedure, bone marrow aspirations were done which resulted in normal findings. The trucut biopsy of the thoracal mass resulted as small round blue malignant tumor cells consistent with peripheral neuroectodermal tumor/ewing sarcoma which was very rare in this age group.

\section{VALUE OF BLOOD BIOMARKERS TO IDENTIFY YOUNG FEBRILE INFANTS DIAGNOSED WITH UTI AT HIGHER RISK FOR BACTEREMIA. INITIAL RESULTS}

doi:10.1136/archdischild-2012-302724.1475

${ }^{1} \mathrm{R}$ Velasco, ${ }^{2} \mathrm{JE}$ Trujillo, ${ }^{2} \mathrm{H}$ Benito, ${ }^{3} \mathrm{~B}$ Serrano, ${ }^{4} \mathrm{~B}$ Gomez, ${ }^{5} \mathrm{M}$ de la Torre, ${ }^{4} \mathrm{~S}$ Mintegi, RISEUP-SPERG. 'Pediatric Emergencies; ${ }^{2}$ Pediatrics, Hospital Rio Hortega, Valladolid; ${ }^{3}$ Preventive Medicine and Epidemiology, Hospital Clinic-Idibaps, Barcelona; ${ }^{4}$ Pediatric Emergencies, Hospital de Cruces, Barakaldo; ${ }^{5}$ Pediatric Emergencies, Hospital Niño Jesus, Madrid, Spain 
Introduction Selected young febrile infants with urinary tract infection (UTI) and lower risk for associated bacteremia can be managed as outpatients. Different biomarkers are useful in the management of febrile young infants but their role in this specific population has not been clarified.

Objective To analyze the relationship between blood biomarkers (white blood cell count -WBC-, absolute neutrophil count -ANC-, procalcitonin -PCT- and C-reactive protein -CRP) and the risk of bacteremia in infants under three months with febrile UTI.

Patients and Methods Prospective multicentric study developed in 29 Spanish Paediatric Emergency Departments members of the RISEUP-SPERG (Spanish Pediatric Emergency Research Group), including febrile infants less than three months old diagnosed with UTI between October-2011 and March-2012.

Results We included 715 infants, being 151 (21.1\%) diagnosed with UTI. Forty-five (28.6\%) were excluded because not having obtained CRP or PCT. Finally, 106 febrile infants under three months diagnosed with UTI were included (5-4.7\%- with associated bacteremia). Values of WBC and ANC were similar in infants with and without bacteremia (14116 leucocytes $/ \mathrm{mm}^{3}$, CI 95\% 11178-17053 vs 15630 , CI 95\% 14221-17039; and 8912 neutrophils $/ \mathrm{mm}^{3}$, CI 95\% 4865-12960 vs 8351, CI 95\% 7327-9375; respectively). Values of CRP and PCT were significantly lower in patients without bacteremia when compared with those with bacteremia $(107.7 \mathrm{mg} / \mathrm{L}, \mathrm{CI}$ 95\% 60.1-155.3; vs 48.8, CI95\% 37.9-59.7; and 26.3, CI 95\% 6.845.9 vs 2.6 , CI 95\% $1.2-4.0$ )

Conclusion Procalcitonin and C-reactive protein identify better than classical biomarkers young febrile infants with UTI at higher risk for bacteremia.

\section{REDUCED CONTAMINATION OF BLOOD CULTURES DRAWN IN THE ED. IMPACT OF THE IMPLEMENTATION OF AN EXTRACTION PROTOCOL}

doi:10.1136/archdischild-2012-302724.1476

'R Velasco, ' JL Fernandez, 'N Campo, 'S Puente, ${ }^{2} \mathrm{M}$ Dominguez-Gil, 'M Sanchez,

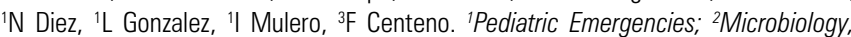
${ }^{3}$ Pediatrics, Hospital Rio Hortega, Valladolid, Spain

Introduction Blood culture is the gold standard for the diagnosis of bacteremia. Clinical guidelines consider adequate a contamination rate of less than 3\%. Between 2009 and 2010 in our service this rate was $12^{\prime} 56 \%$. Therefore, in February-2011 we started a protocol for blood cultures according to the Scientific Evidence.

Objectives Analyze the impact of implementing a protocol for the extraction of hemocultures on contamination rates.

Methods Retrospective study cohort: Pre-implantation group (A): Patients whom a blood culture was taken from in our Pediatric Emergency Department between March 2009 and April 2010 and post-implantation group (B): Patients with blood culture between April 2011 and March 2012.

Results In the group A 1098 blood cultures were obtained (4'08\% of 26874 patients attended) and in group B 535 (2'87\% of 18628 patients, $\mathrm{p}=0.0001)$. A true pathogen was isolated in 11 samples in cohort A $\left(1^{\prime} 00 \%\right.$, CI95\% $0.41-1.59 ; 0.04 \%$ of the population attended from CI $95 \% 0.02$ to 0.06$)$ and in 9 of the group B (1'68\%; CI $95 \% 0.59-2.77 ; 0$; $048 \%$ of the population attended CI $95 \% 0.02$ to 0.08$)$.

In the group A germs considered contaminants grew in 138 blood cultures $\left(12^{\prime} 56 \%\right.$, CI $\left.95 \% 10.6-14.52\right)$ and in 34 in the B (6'35\%, C195\%: 4.28-8.42).

Conclusion The development of a protocol for blood cultures extraction technique according to the Scientific Evidence is useful to reduce sample contamination, but it didn't reach to the standards of quality. Technique protocolization was associated with a significant decrease in number of blood cultures drawn.

\section{PROGNOSTIC VALUE OF INITIAL ARTERIAL LACTATE LEVELS OF ACUTE CARBON MONOXIDE POISONING IN CHILDREN}

doi:10.1136/archdischild-2012-302724.1477

S Sahin. Eskisehir Osmangazi University, Eskisehir, Turkey

Aim The present study was aimed to compare the prognostic values of lactate and carboxyhemoglobin levels on clinical neurological and cardiac involvement in children with carbon monoxide poisoning.

Methods Totally 77 children aged to 1 month and 17 years who referred to Paediatric Emergency Department (PED) between 1 April 2009 and 30 April 2011 and diagnosed with carbon monoxide intoxication were assessed for age, gender, symptoms, clinical findings, blood gas lactate levels, carboxyhemoglobin levels, morbidity and mortality.

Results There was a significant positive correlation between initial lactate levels in blood gas analysis and early and late complications, neurological and cardiac involvements and morbidity compared to carboxyhemoglobin levels.

Discussion The initial lactate levels have higher prognostic value for cardiac involvement and late neurological sequelae compared to carboxyhemoglobin and it may be helpful for treatment.

\begin{tabular}{l}
\hline 1478 ATTENDANCES AT THE CHILDREN'S EMERGENCY \\
DEPARTMENT: AN AUDIT OF TWO YEARS OF ACTIVITY \\
AT A NEW, DEDICATED PAEDIATRIC EMERGENCY \\
DEPARTMENT
\end{tabular}

doi:10.1136/archdischild-2012-302724.1478

1,2R Isba, ${ }^{2} \mathrm{M}$ Gibb, 'AG Rowland. 'Emergency Department, North Manchester General Hospital, Manchester; ${ }^{2}$ Medical School, Lancaster University, Lancaster, UK

Aim The aim of this audit was to explore patterns of attendance at a new, dedicated, audio-visually separate paediatric emergency department (PED) within the ED of a hospital in the North West of England. The PED was opened in June 2009 following the major restructuring of paediatric services in Manchester.

Methods This was a retrospective audit of paediatric attendances (those aged < 16 years) to the ED of North Manchester General Hospital between June 2009 and June 2011.

Results During the first year there were 26073 attendances to the PED, while in the second there were 24119 (just over a quarter of all attendances to the ED). More than half of all attendees were in the pre-school age group. There were low levels of attendance overnight, and numbers of attendees peaked around $6 \mathrm{pm}$. Mondays were the busiest days (mean attendees 75.4) and Fridays the quietest (mean attendees 64.6). Whilst the average length of stay was just over two hours, this varied depending on the time of arrival. Approximately one in six children was referred to an inpatient specialty for further assessment.

Conclusion These results have implications for those managing EDs that care for children. Knowledge of attendance patterns may enable managers to better plan staffing levels and deployment within an ED, and there are wider implications for the hospital as a whole. By gaining a better understanding of the patterns of attendance it may be possible to predict future patterns (e.g. via mathematical modelling), and to plan resources accordingly.

\section{REFERRAL OF CHILDREN FROM A UK DISTRICT GENERAL HOSPITAL EMERGENCY DEPARTMENT TO PRIMARY CARE GENERAL PRACTITIONERS}

doi:10.1136/archdischild-2012-302724.1479

${ }^{1} \mathrm{AG}$ Rowland, ${ }^{1,2} \mathrm{R}$ Isba. 'Emergency Department, North Manchester General Hospital, Manchester; ${ }^{2}$ Medical School, Lancaster University, Lancaster, UK 